
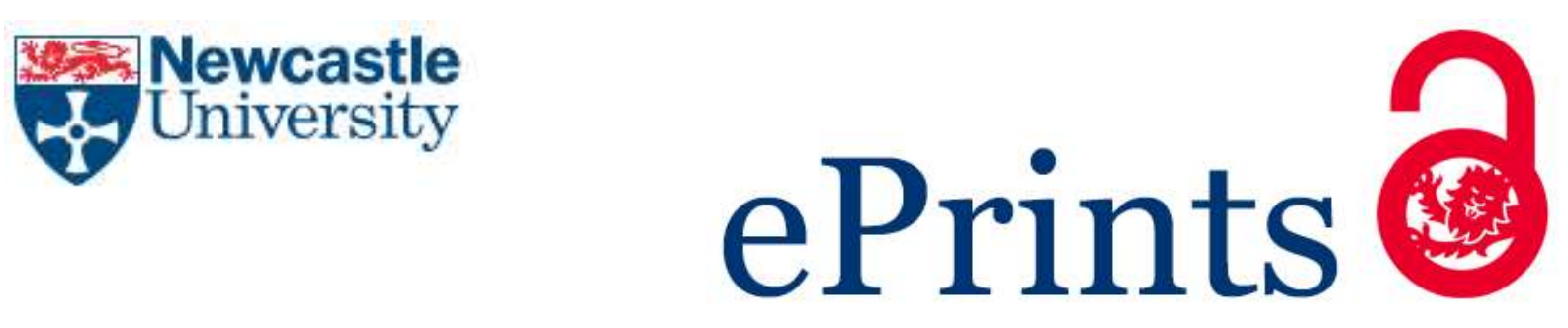

Teo TT, Logenthiran T, Woo WL, Abidi K. Fuzzy logic control of energy storage system in microgrid operation. In: 2016 IEEE Innovative Smart Grid Technologies - Asia (ISGT-Asia). 2016, Melbourne: IEEE.

\title{
Copyright:
}

(C) 2016 IEEE. Personal use of this material is permitted. Permission from IEEE must be obtained for all other uses, in any current or future media, including reprinting/republishing this material for advertising or promotional purposes, creating new collective works, for resale or redistribution to servers or lists, or reuse of any copyrighted component of this work in other works.

DOI link to article:

https://doi.org/10.1109/ISGT-Asia.2016.7796362

Date deposited:

$21 / 03 / 2017$ 


\section{Fuzzy Logic Control of Energy Storage System in Microgrid Operation}

\author{
T.T. Teo
}

School of Electrical and Electronic Engineering Newcastle University, Singapore
T. Logenthiran

School of Electrical and

Electronic Engineering

Newcastle University,

Singapore
W. L. Woo

School of Electrical and

Electronic Engineering

Newcastle University,

Singapore
K. Abidi

School of Electrical and

Electronic Engineering

Newcastle University,

Singapore

t.t.teo@newcastle.ac.uk t.logenthiran@newcastle.ac.uk lok.woo@newcastle.ac.uk khalid.abidi@newcastle.ac.uk

\begin{abstract}
Recent development in Renewable Energy Sources (RES) have led to a higher penetration in existing power systems. As the majority of RES are intermittent by nature, it presents major challenges to the grid operators. An Energy Storage System (ESS) can be connected to mitigate this intermittent sources. When multiple renewable energy sources, flexible loads and ESS are connected to the grid, complexity of the system is significantly increased. Such problems have multiple constraints and objective hence it is challenging to design an effective rulebased control strategy. A control strategy based on fuzzy logic which is similar to human reasoning tolerates uncertainties and imprecision. The proposed fuzzy inference system (FIS) aims to reduce the grid fluctuation and increase the energy storage lifecycle by deciding when and how much to charge/discharge the ESS. A real data was used to test and validate the proposed FIS.
\end{abstract}

In this paper, MATLAB/Simulink is used to create and implement the microgrid test bench and FIS. The proposed methodology is tested against a rule-based control strategy. Simulation studies were carried out on the developed model and results have shown that the proposed FIS can effectively reduce the fluctuation and prolong the life cycle of the ESS.

Index Terms-Energy Storage System, Fuzzy Inference System, Energy Management System, Battery Life Cycle, Microgrid

\section{INTRODUCTION}

The world energy demand is increasing steadily over the years. With the finite amount of fossil fuels and raising concern of its negative environmental impacts has led to a higher penetration of Renewable Energy Sources (RES) in existing power system. Majority of the power produced by RES are intermittent in nature hence impact the balancing of the grid.

Large scale photovoltaic (PV)/ wind farm can be operated from a centralized location by the grid operators while a smaller scale can be operated by the consumers. The former can be controlled via a centralized controller while the latter requires a decentralized controller.

Energy Management System (EMS) has been applied to the process of monitoring, controlling, and conserving energy in a building, organization, or distribution system [1] . It comprises of a number of key functions to match supply with demand, handle disturbances and optimizations all within the operation constraints. The typical objectives of EMS are minimizing energy usage, energy cost and environmental impacts. EMS can focus on single objective optimization or multiple objectives optimization.

- Integrating Energy Storage and optimize life cycle

- Minimizing peak load and fluctuation on grid

- Minimizing operating cost

The fundamental objective of grid operation is balancing the supply and demand at all time. The grid operators have to anticipate when there will be a surplus or shortage in power production.

There is a growing interest in smaller type of power system called Microgrid, which usually comprises of local generation and control capability. It operates either in grid-connected or islanded mode [2]. The former is capable of reducing the demand from main grid. The latter is isolated and expected to be able to supply at least a portion of the demand connected in an event of power outages and remain operational in this mode. The time taken to setup a microgrid is significantly lesser than large centralized power plants. It can be deployed in rural areas which are far from large centralized power plants.

When RES, ESS, conventional generation and changing load are connected to the power system, complexity of the system significantly increased. Such problems have multiple constraints and sometimes contradicting objectives, it can be difficult to design an effective control strategy.

Distributed generation (DG) unit is usually smaller compared to a centralized generation unit and it is located closer to the load. DG includes PV, wind, combine heat and power (CHP) plants, micro turbine and storage systems. It introduces many potential benefits such as enhanced reliability and green power [3]. There are several challenges when a significant number of DG is connected due to [4]:

- Computational burden due to the number of DG units and loads

- Lack of dedicated management unit 
- Frequent redesign when there is a change in configuration

- Reliability and security vulnerability due to common point of failure

Recent developments in smart grid technologies have led to a renewed interest in using Fuzzy Logic to take on several tasks of EMS such as forecasting of RES/load [5] and maximum power point tracking (MPPT) control of solar power [6].

An effective control strategy must be used to fully benefit from integrating Energy Storage System (ESS). A Fuzzy Inference System (FIS) controller is proposed in this paper to determine the charging/discharging rate of the ESS depending on the RES and current State-of-Charge (SOC) of the ESS.

The rest of this paper is organized as follow: Section II provides a background information on management of ESS and presents a storage management strategy to compare with the proposed methodology. Section III presents the proposed methodology and quality indices used to measure its effectiveness. Section IV presents the ratings of the microgrid test bench, simulation parameters and results. A detailed comparison between the effectiveness of the proposed fuzzy controller and a rule-based controller is presented in Section $\mathrm{V}$. The paper is concluded in Section VI.

\section{BACKGROUND INFORMATION}

Energy Storage System can be connected to the system to store surplus energy when supply is greater than demand and use it when supply is lesser than demand. The applications greatly rely on the characteristic of ESS. Some application requires fast charging/discharging rate while other requires a larger capacity. A larger capacity ESS is required for backup/emergency supply while a faster charging/discharging rate is required to reduce fluctuation of RES. The combination of different types of RES and ESS can complement each other and improve the system reliability [7].

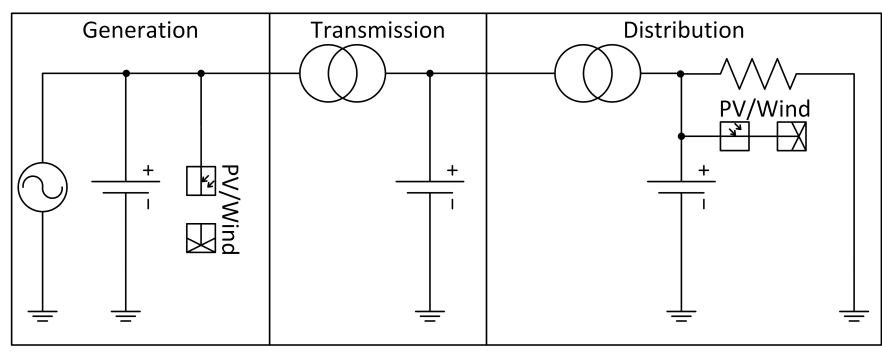

Fig. 1. Energy Storage System at different Network location

Fig. 1 illustrates a simplified modern power system where ESS and RES can be installed on any location in the system. At generation/distribution side, it can reduce fluctuation of intermittent generation of RES [8] and it can reduce transmission congestion [9].
Intuitively, ESS charges when the demand is below the supply and discharges when it is above. This can be useful to keep the transmission lines within its limits and avoid the use of peak load generators [10].

For emergency supply, ESS charges to its maximum capacity and remains at it and only discharge to continuously supply the load in an event of power outage or restart the system after a complete blackout. ESS can also reduce the fluctuation of the intermittent generation of RES [11], [12]. Different applications of ESS requires a different control strategy.

From Fig. 1, distribution side shows the test system configuration used in this paper, where energy storage system and renewable energy sources are connected to the load.

$P_{\text {res }}$ is the renewable power generated from the two RES connected. $P_{\text {balance }}$ is the surplus/shortage of power between $P_{\text {load }}$ and $P_{\text {res }}$ which can be met by charging/discharging ESS, $P_{\text {ess }} . P_{\text {balance }}$ also correspond to the power profile without an ESS. The remaining power is absorbed/injected by/to the grid, $P_{\text {grid }}$. The objective is to minimize the fluctuation of $P_{\text {grid }}$.

$$
\begin{aligned}
& P_{\text {res }}=P_{p v}+P_{\text {wind }} \\
& P_{\text {balance }}=P_{\text {load }}-P_{\text {res }} \\
& P_{\text {grid }}=P_{\text {balance }}+P_{\text {ess }}
\end{aligned}
$$

State-of-Charge (SOC) represents the current capacity of the ESS with respect to its full capacity in percentage; $0 \%$ is fully discharge and $100 \%$ is fully charged. The SOC can be estimated from (5). Capacity fade refers to the loss of discharging capacities over time and life cycle refers to the number of charging/discharging cycles before reducing its capacity to $80 \%$ of its initial capacity[13]. A four-fold increase in life cycle when the SOC is above $50 \%$ compared to $0 \%$ [14]. By limiting the maximum and minimum threshold can increase the life cycle at the expense of not fully utilizing it.

The rating of ESS is expressed in $(\mathrm{kWh})$, therefore SOC of ESS can be expressed as

$$
S O C_{e s s}(n)=\frac{E_{e s s}(n)}{E_{e s s, \max }}
$$

Where $S O C_{e s s}(n)$ is the current capacity of ESS, $E_{e s s, \max }$ is the maximum capacity and $E_{\text {ess }}(n)$ is the current capacity of ESS.

$$
E_{\text {ess }}(n)=E_{\text {ess }}(n-1)+\Delta E_{\text {ess }}(n)
$$

From (6), $\Delta E_{\text {ess }}(n)$ indicates charging/discharging of ESS. There are several numerical integration methods to esti- 
mate $\Delta E_{e s s}(n)$ from 5 . The method used in this paper is Rectangle rule.

$$
\begin{aligned}
& \text { Rectangle rule, } \int_{a}^{b} f(t) d t=f(a) \cdot(b-a) \\
& \Delta E_{\text {ess }}(n)=\int_{(n-1)}^{n} P_{\text {ess }}(n) d n \simeq P_{\text {ess }}(n-1)
\end{aligned}
$$

Fig. 2 shows a Rule-Based Controller (RBC) flow chart which is used to compare with the proposed FIS. This rule-based control strategy will charge ESS when there is a surplus of RES and ESS is not at its full capacity and discharge ESS when there is a lack of RES and ESS is not empty. The charging/discharging rate for this $\mathrm{RBC}$ is a constant $\pm 2 \mathrm{~kW}$.

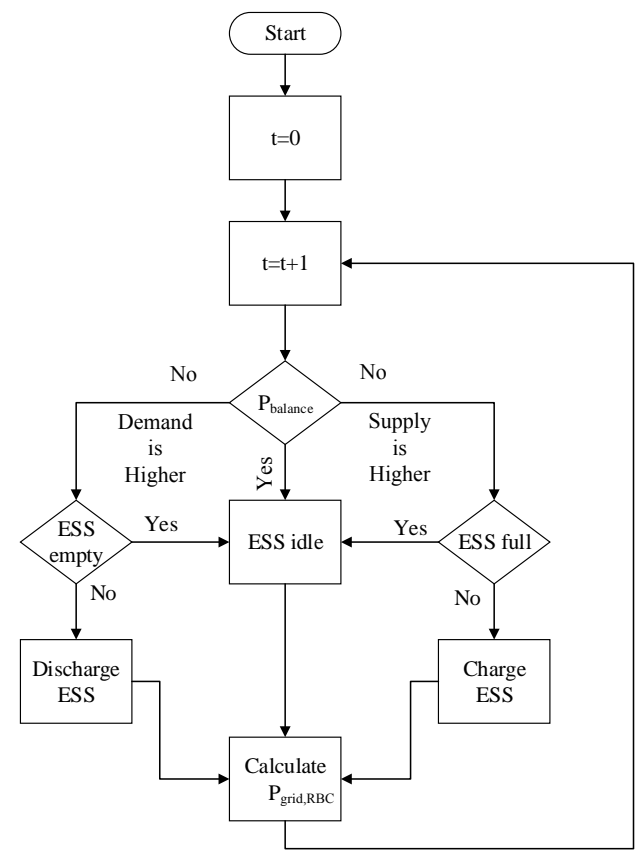

Fig. 2. Rule-based Control for ESS

\section{Proposed Methodology}

This section is divided into two parts; Quality Indices and Fuzzy Controller Design. The first part will explain in details the indices used to measure the effectiveness of the fuzzy controller. The second part will explain in details the design process for the fuzzy controller.

\section{A. Quality Indices}

To evaluate the effectiveness of the proposed fuzzy controller, a set of indices based on power variation range (PVR), power quality (PQ) and battery dynamic range (BDR) as proposed in [15]. These indices can quantify the effectiveness of various EMS objectives.
1) Power Variation Range(PVR):

$$
P V R=\frac{\left|P_{\text {grid,max }}\right|+\left|P_{\text {grid,min }}\right|}{\left|P_{\text {balance, } \max }\right|+\left|P_{\text {balance, } \text { min }}\right|}
$$

Where the numerator and denominator are the sum of maximum and minimum value from $P_{\text {grid }}$ and $P_{\text {balance }}$ respectively. $P V R<1$ indicates a reduction in variation in the $P_{\text {grid }}$ and $P V R>1$ indicates more variation than without an ESS and should be avoided. This index should be minimized.

2) Power Quality $(P Q)$ :

$$
P Q=\frac{\sqrt{\frac{1}{m} \sum_{n=2}^{\infty} P_{\text {grid }}^{2}(n)}}{P_{\text {grid,mean }}}
$$

Where $P_{\text {grid }}(n)$ is the $n^{\text {th }}$ harmonic component in $P_{\text {grid }}$ from the Fast-Fourier-Transform (FFT) deposition, $P_{\text {grid,mean }}$ is the yearly average power and $m$ is the number of samples. A small value of PQ indicates the absence of higher harmonics component. This index should be minimized.

3) Battery Dynamic Range (BDR):

$$
B D R=E_{e s s, \max }-E_{e s s, \min }
$$

Where BDR is the maximum changes in ESS over the course of one year. Over discharging the ESS has negative impact on its life cycle. This index should be minimized as well.

\section{B. Fuzzy Controller Design}

Fuzzy Inference System (FIS) can handle imprecise or vague information. The general diagram of a FIS is shown in Fig. 3. A Mamdani-type FIS is used in this paper.

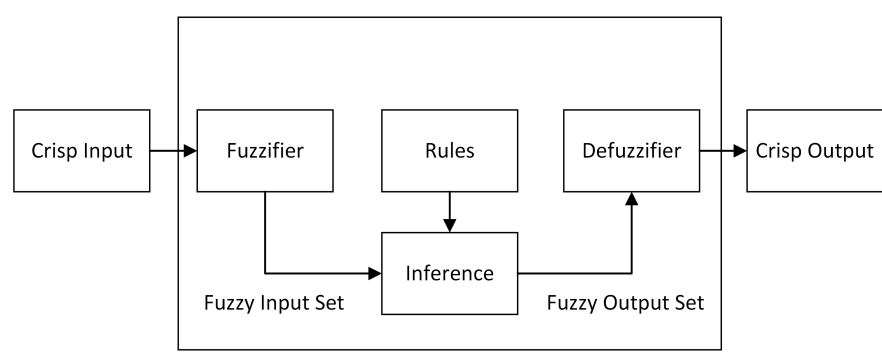

Fig. 3. General Diagram of FIS

1) Fuzzifier: is the process where crisp inputs or real inputs are mapped to fuzzy input using membership function. Membership function (MF) introduces nonlinearity to the input(s). The system's designer chooses the membership function. The input/output MF for $P_{\text {balance }}, S O C_{\text {ess }}$ and $P_{\text {ess }}$ are assumed to be five uniformly distributed triangular MF along its respective range as shown in Fig. 4. 


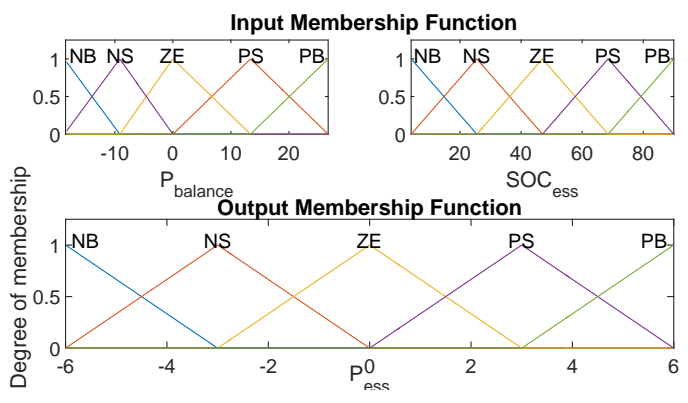

Fig. 4. Input/Output Membership Function

2) Rules: are a set of linguistic statements based on a collection of IF-THEN statement, which are typically based on human expert knowledge. It is easy to interpret these rules as they are highly similar to natural human language. The effectiveness of FIS is proportional to the knowledge of the designer. The rules in this paper is set based on the following:

- The ESS should charge/discharge when $P_{r e s}$ is higher/lower than $P_{\text {load }}$

- ESS should maintain a SOC above 5\% to prevent overdischarging

The objective and constraints can be described as:

Minimize $P_{\text {grid }}$

Subject to

$$
\begin{aligned}
& P_{\text {balance,min }} \leq P_{\text {balance }} \leq P_{\text {balance, } \text { max }} \\
& S O C_{\text {ess }, \text { min }} \leq S O C_{\text {ess }} \leq S O C_{\text {ess }, \text { max }} \\
& P_{\text {ess,min }} \leq P_{\text {ess }} \leq P_{\text {ess,max }}
\end{aligned}
$$

These constraint are taken into consideration when designing the membership function of the FIS.

The first constraint is maximum and minimum values of the difference between the load and RES. The second constraint is the maximum and minimum SOC threshold of the ESS. The last constraint is the maximum and minimum allowable charging/discharging rate for the ESS.

The FIS will work towards the objective of minimizing variation in the grid while satisfying all the constraint. A total of 25 rules are set based on expert knowledge and shown in Table I. The $5 \mathrm{MF}$ of Table I represents the output of $P_{\text {ess }}$. Where N, P, B, S and ZE represents Negative, Positive, Big, Small and Zero respectively.

TABLE I

FUZZY RULES

\begin{tabular}{|l|l|c|c|c|c|c|}
\hline \multicolumn{2}{|c|}{$P_{\text {ess }}$} & \multicolumn{5}{c|}{$P_{\text {balance }}$} \\
\cline { 3 - 7 } & & NB & NS & ZE & PS & PB \\
\hline \multirow{4}{*}{ SOC $_{\text {ess }}$} & NB & PB & PS & ZE & ZE & ZE \\
& NS & PB & PS & ZE & NS & NS \\
& ZE & PS & PS & ZE & NS & NS \\
& PS & PS & PS & ZE & NS & NB \\
& PB & ZE & ZE & ZE & NS & NB \\
\hline
\end{tabular}

The highlighted cell from Table I corresponds to the rule:

IF $P_{\text {balance }}$ is NB and SOCess is NB THEN $P_{\text {ess }}$ is PB

When $P_{\text {balance }}$ is NB, it means $P_{\text {load }} \ll P_{\text {res }}$ and $S O C_{\text {ess }}$ is NB, it means ESS is near its minimum threshold, therefore $P_{e s s}$ is PB indicates to charge the ESS at a higher rate.

$$
P_{\text {balance }}= \begin{cases}P_{\text {load }}>P_{\text {res }} & \text { if } 0 \leq P_{\text {balance }} \\ P_{\text {load }}=P_{\text {res }} & \text { if } P_{\text {balance }}=0 \\ P_{\text {load }}<P_{\text {res }} & \text { if } P_{\text {balance }} \leq 0\end{cases}
$$

$$
S O C_{\text {ess }}= \begin{cases}\text { Idle } & \text { if } S O C_{\text {ess }} \leq 4 \\ & \text { or } 90 \leq S O C_{\text {ess }} \\ \text { Operating } & \text { if } 4 \leq S O C_{\text {ess }} \leq 90\end{cases}
$$

$$
P_{\text {ess }}= \begin{cases}\text { Disharging } & \text { if } P_{\text {ess }} \leq 0 \\ \text { Idle } & \text { if } P_{\text {ess }}=0 \\ \text { Charging } & \text { if } 0 \leq P_{\text {ess }}\end{cases}
$$

3) Inference: is the process of simulating human decision based on fuzzy concept. It is the mapping from a given input(s) to an output(s).

4) Defuzzifier: There are many defuzzifier proposed in the literature. The defuzzifier used in this paper is centroid. This process will compute a crisp output based on the output of the inference.

A FIS is implemented using MATLAB/Simulink. A simplified diagram is shown in Fig. 5. The FIS inputs are $P_{\text {balance }}$ and $S O C_{e s s, F I S}$ respectively and output is $P_{e s s, F I S}$. P $P_{\text {grid,FIS }}$ can be calculated using Equation (3)

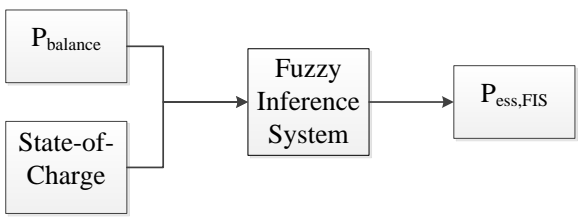

Fig. 5. Simplified Fuzzy Block Diagram

\section{Simulation And Results}

MATLAB/Simulink is used to implement the FIS, RBC and microgrid in order to simulate and verify the effectiveness of the proposed methodology. Three different test cases are studied in this paper.

Case 1: No ESS

Case 2: ESS with FIS

Case 3: ESS with RBC 
Test case 1 is used as a benchmark for Test case 2 and 3. It shows the quality indices without an ESS hence able to verify the efficiency of the proposed control methodology.

Fig. 6 shows a grid-tied domestic microgrid which consist of two renewable energy sources, photovoltaic power, $P_{p v}$ and wind power, $P_{\text {wind }}$ and an energy storage system, $E_{\text {ess }}$. The rating of each sources are $13.68 \mathrm{kWp}, 12 \mathrm{kWp}$ and $90 \mathrm{kWh}$

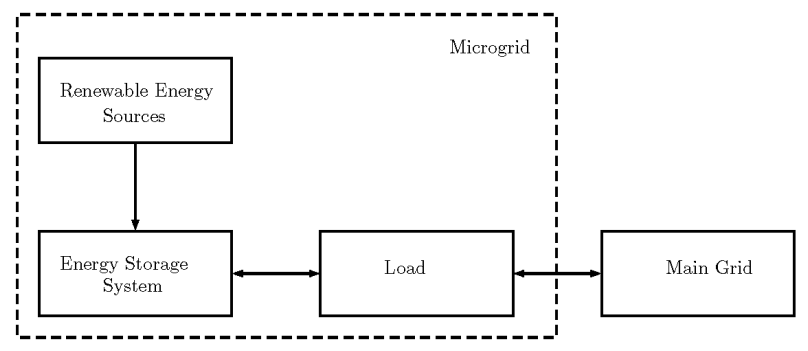

Fig. 6. Grid-Tied Domestic Microgrid

respectively. The load, $P_{\text {load }}$ has a rated power of $26.8 \mathrm{kWp}$. The rating of each element of the microgrid is shown in Table II. The data used in this simulation are obtained from [16] and sampled at every 15 minute for a year. The resulting dataset consist of 35040 data points for each parameters.

The result presented in this section shows the first two day of the data.

TABLE II

PARAMETERS OF TEST SYSTEM

\begin{tabular}{|l|l|}
\hline Parameter & Rating \\
\hline PV Array & $13.68 \mathrm{kWp}$ \\
Wind Turbine & $12 \mathrm{kWp}$ \\
Energy Storage System & $90 \mathrm{kWh} / 6 \mathrm{~kW}$ \\
Load & $26.8 \mathrm{kWp}$ \\
\hline
\end{tabular}

Fig. 7 shows $P_{\text {res }}$ and $P_{\text {load }}$ respectively. The output power profile of $P_{\text {res }}$ experienced many peaks and troughs throughout the day. Hence integrating power sources with such high intermittent nature might have adverse impact to the power grid. It emphasize on the importance of an effective controller to fully utilize RES and ESS.

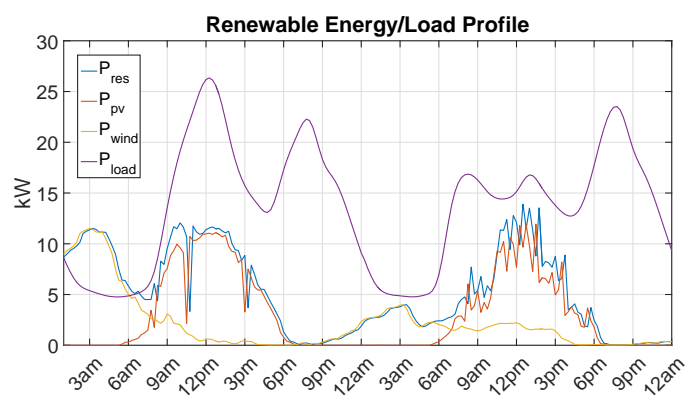

Fig. 7. Profile of RES

From Fig. 8, the power profile of RBC, $P_{\text {grid, } R B C}$ shows that there is a $\pm 2 \mathrm{~kW}$ offset from $P_{\text {balance. }}$. There are multiple instances where $\mathrm{RBC}$ charge/discharge more than required (highlighted in red).

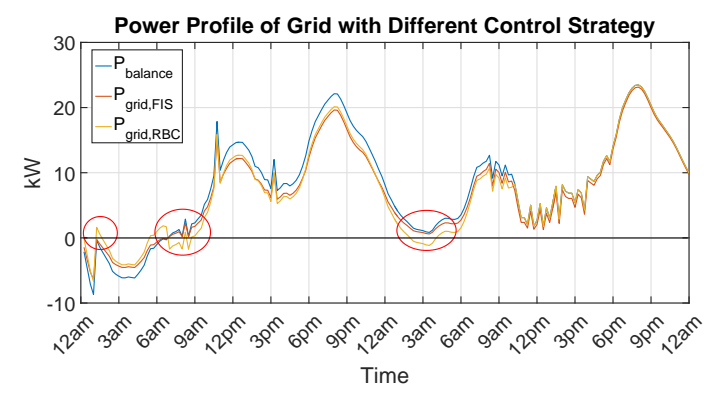

Fig. 8. Comparison between $P_{\text {balance }}, P_{\text {grid,FIS }}$ and $P_{\text {grid,RBC }}$

By discharging more than required by the grid will reduce the SOC to its minimum capacity prematurely as shown in Fig. 9. The ESS is unable to contribute any further after it reaches the maximum/minimum capacity hence render it useless.

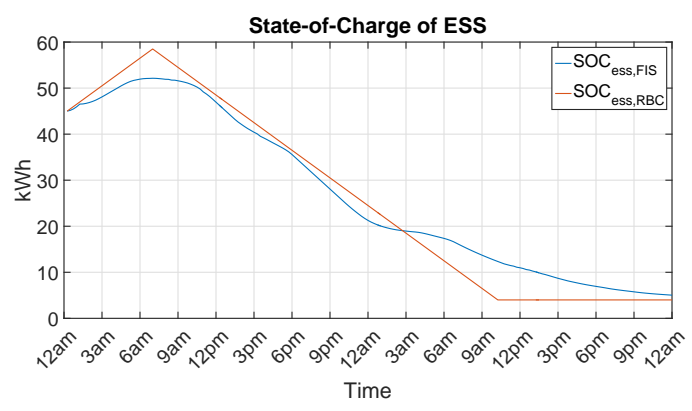

Fig. 9. Comparison of $S O C_{\text {ess }}$ between FIS and RBC

Fig. 10 shows the charging/discharging of ESS. The FIS is able to charge/discharge at a higher/lower rate hence able to match the supply and demand better while RBC can only charge/discharge at a constant rate. For instance, when $P_{\text {balance }}<2 k W$, by discharging the ESS at $2 \mathrm{~kW}$ will introduce excess power which must be met by the grid.

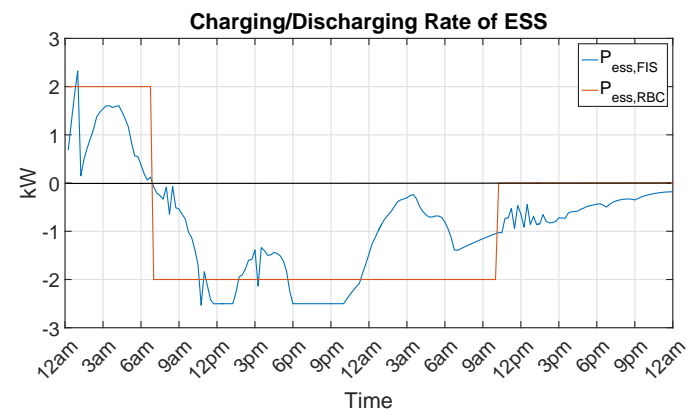

Fig. 10. Charging/Discharging rate of ESS 


\section{DISCUSSION}

PVR is reduced in $P_{\text {grid,FIS }}$ indicates the proposed FIS is effective. Table III presents a summary of the Quality Indices used in this paper for various control strategy.

TABLE III

QUALITY INDICES

\begin{tabular}{|l|c|c|c|c|c|}
\hline Case & PVR & $P_{\text {grid, } \text { min }}$ & $P_{\text {grid, } \max }$ & PQ & BDR \\
\hline 1 & 1 & -16.744 & 26.73 & 1.2344 & - \\
\hline 2 & 0.9277 & -13.657 & 26.67 & 1.1576 & 83.72 \\
\hline 3 & 0.9540 & -14.744 & 26.73 & 1.1412 & 86 \\
\hline
\end{tabular}

The results clearly indicate that the variation of power profile is reduced when using FIS and RBC. When using FIS, the maximum and minimum power of the grid are reduced while only the minimum power is reduced in RBC. In this multiple objective/constraint problem, the control strategy have to handle imprecise and vague information.

While the RBC shows satisfactory performance by reducing the variation of the grid profile, this can be further improve by using a FIS. As the prices of ESS continues to decline with advances in technologies, the lower prices which makes it attractive for grid-operator and home owner to implement.

\section{CONCLUSION AND FUTURE WORK}

ESS can increase reliability and resiliency of the microgrid only if the control strategy is effective. The proposed methodology controls the charging/discharging rate has shown good result. This is done by considering multiple objectives and constraints. The fuzzy controller proposed in this paper has effectively reduce the variation of $P_{\text {grid }}$ and prevent over-discharging of the ESS which deteriorate its life span. The comparison between a microgrid with and without ESS has demonstrated that an effective controller can result in better power quality and increase the life cycle simultaneously.

Future work include forecasting [17] as one of the inputs for FIS and integrate with an expendable and adaptable MultiAgent-System to be part of a more robust and complete EMS [18].

\section{REFERENCES}

[1] H. A. Mostafa, R. E. Shatshat, and M. M. A. Salama, "A review on energy management systems," in 2014 IEEE PES T D Conference and Exposition, April 2014, pp. 1-5.

[2] D. E. Olivares, A. Mehrizi-Sani, A. H. Etemadi, C. A. Caizares, R. Iravani, M. Kazerani, A. H. Hajimiragha, O. Gomis-Bellmunt, M. Saeedifard, R. Palma-Behnke, G. A. Jimnez-Estvez, and N. D. Hatziargyriou, "Trends in microgrid control," IEEE Transactions on Smart Grid, vol. 5, no. 4, pp. 1905-1919, July 2014.
[3] P. Dondi, D. Bayoumi, C. Haederli, D. Julian, and M. Suter, "Network integration of distributed power generation," Journal of Power Sources, vol. 106, no. 12, pp. 1 - 9, 2002, proceedings of the Seventh Grove Fuel Cell Symposium. [Online]. Available: http://www.sciencedirect.com/science/article/pii/S037877530101031X

[4] M. Yazdanian and A. Mehrizi-Sani, "Distributed control techniques in microgrids," IEEE Transactions on Smart Grid, vol. 5, no. 6, pp. 29012909, Nov 2014

[5] D. Sez, F. vila, D. Olivares, C. Caizares, and L. Marn, "Fuzzy prediction interval models for forecasting renewable resources and loads in microgrids," IEEE Transactions on Smart Grid, vol. 6, no. 2, pp. 548-556, March 2015.

[6] C. S. Chiu, "T-s fuzzy maximum power point tracking control of solar power generation systems," IEEE Transactions on Energy Conversion, vol. 25 , no. 4, pp. 1123-1132, Dec 2010.

[7] X. Qiu, T. A. Nguyen, and M. L. Crow, "Heterogeneous energy storage optimization for microgrids," IEEE Transactions on Smart Grid, vol. PP, no. 99 , pp. 1-1, 2015.

[8] M. S. Lu, C. L. Chang, W. J. Lee, and L. Wang, "Combining the wind power generation system with energy storage equipment," IEEE Transactions on Industry Applications, vol. 45, no. 6, pp. 2109-2115, Nov 2009.

[9] L. S. Vargas, G. Bustos-Turu, and F. Larran, "Wind power curtailment and energy storage in transmission congestion management considering power plants ramp rates," IEEE Transactions on Power Systems, vol. 30 no. 5, pp. 2498-2506, Sept 2015.

[10] B. J. Donnellan, D. J. Vowles, and W. L. Soong, "A review of energy storage and its application in power systems," in Power Engineering Conference (AUPEC), 2015 Australasian Universities, Sept 2015, pp. $1-6$.

[11] F. Marra, G. Yang, C. Trholt, J. stergaard, and E. Larsen, "A decentralized storage strategy for residential feeders with photovoltaics," IEEE Transactions on Smart Grid, vol. 5, no. 2, pp. 974-981, March 2014.

[12] D. Arcos-Aviles, J. Pascual, L. Marroyo, P. Sanchis, F. Guinjoan, and M. P. Marietta, "Optimal fuzzy logic ems design for residential gridconnected microgrid with hybrid renewable generation and storage," in 2015 IEEE 24th International Symposium on Industrial Electronics (ISIE), June 2015, pp. 742-747.

[13] R. Spotnitz, "Simulation of capacity fade in lithiumion batteries," Journal of Power Sources, vol. 113 , no. 1, pp. $72-80, \quad 2003$. [Online]. Available: http://www.sciencedirect.com/science/article/pii/S0378775302004901

[14] T. Guena and P. Leblanc, "How depth of discharge affects the cycle life of lithium-metal-polymer batteries," in Telecommunications Energy Conference, 2006. INTELEC '06. 28th Annual International, Sept 2006, pp. 1-8.

[15] D. A. Avils, F. Guinjoan, J. Barricarte, L. Marroyo, P. Sanchis, and H. Valderrama, "Battery management fuzzy control for a grid- tied microgrid with renewable generation," in IECON 2012 - 38th Annual Conference on IEEE Industrial Electronics Society, Oct 2012, pp. 5607 5612.

[16] C. Draxl, B. Hodge, A. Clifton, and J. McCaa, "Overview and meteorological validation of the wind integration national dataset toolkit," NREL/TP-5000-61740. Golden (CO): National Renewable Energy Laboratory (forthcoming), Tech. Rep., 2015.

[17] T. T. Teo, T. Logenthiran, and W. L. Woo, "Forecasting of photovoltaic power using extreme learning machine," in Smart Grid Technologies Asia (ISGT ASIA), 2015 IEEE Innovative, Nov 2015, pp. 1-6.

[18] T. Logenthiran, "Multi-agent system for control and management of distributed power systems," Ph.D. dissertation, 2012 